\title{
A K-Means Based Multi-level Text Clustering Algorithm for Retrieval of Research Information
}

\author{
Damaris Ndinda Waema \\ Department of Computing \\ Jomo Kenyatta University of \\ Agriculture and Technology \\ Juja, Kenya
}

\author{
Petronilla Muriithi \\ Department of Computing \\ Jomo Kenyatta University of \\ Agriculture and Technology \\ Juja, Kenya
}

\author{
George Okeyo \\ School of Computer Science \\ and Informatics \\ De Montfort University \\ Leicester, United Kingdom
}

\begin{abstract}
Academic researchers in institutions of higher learning and research institutes use research outputs and metadata throughout their research work and to help in identifying research collaborators as well as getting to know existing research. Research outputs range from academic theses, journal and conference articles, books and book chapters, and datasets while research meta-data includes authors, affiliations, research areas, and projects, among others. However, access and retrieval of relevant research outputs and metadata remains a major challenge. As a result there is duplication of research, fewer opportunities for networking, and difficulty in detecting scientific fraud. Efforts need to be made to make academic research outputs and meta-data readily available and easy to retrieve. The main purpose of this work is to develop a tailor-made approach to information retrieval for the retrieval of research information and related meta-data. Therefore, the paper presents a multi-level text clustering algorithm for retrieval of scholarly research outputs and metadata from a central repository through a web based interface. The algorithm first clusters SQL data records that represents meta-data at the first level, then retrieves and clusters text documents representing research outputs at the second level. The algorithm was tested on retrieving information in the areas of text clustering, cloud computing, banking, HIV/AIDS, food security and cancer. The results show that it enables researchers to retrieve relevant information according to their information needs. To enable further enhancements and improvements, the algorithm will be released to the public domain for use in similar application domains or extension by other researchers.
\end{abstract}

Keywords: Text Clustering, Multi-level, Research Metadata, Information Retrieval, SQL Data Clustering

\section{INTRODUCTION}

In order to fuel research activities, it is important to have research data available and easy to retrieve. In the context of this paper, research data refers to research outputs such as journal articles, research papers, and theses produced by postgraduate students. Research data also includes research metadata found in scholarly document repositories such as bio data about researchers or authors, research projects, as well as funding opportunities provided by various funding bodies to provide funds for researchers in their research endeavours. With the availability of reliable communication and networking platforms, access to this research data enables researchers to find other researchers from their research institutions or other institutions with similar research interests whom they can collaborate with. According to Muriithi [1], collaboration among researchers has various advantages such as availability of diverse range of skills, access to resources and special equipment not locally available, higher productivity, and increased visibility of the researchers' research outputs. Xia, Wang, Bekele and Liu [2] also note that it may be difficult to achieve scientific success without collaboration among scholars. Availability and easy retrieval of academic research data also helps to understand the knowledge creation process [3], reduce duplication of research in various research institutions, as well as aiding in curbing scientific fraud.

Availability and access to research data remains a challenge for Kenyan researchers. There does not exist a single national scholarly document repository that consolidates research data from scholars across Kenyan institutions of higher learning as well as research institutions and make it available for retrieval by interested researchers [4]. In a bid to solve this problem, some Kenyan universities have developed their own institutional repositories where they keep research outputs and research metadata from scholars in their universities and allow those researchers and their colleagues to have access to it when need arises. Such universities include Dedan Kimathi University of Technology [5]. However, despite these attempts by Kenyan institutions of higher learning to have their own institutional repositories, availability and retrieval of research outputs and research metadata is still a key challenge facing academic researchers in Kenya. This is because some of these institutional scholarly repositories have very low volumes of data available online [5]. Moreover, since these institutional repositories are usually the property of the particular universities, only researchers from those universities are sometimes given credentials to be able to access the repositories. This makes it hard for researchers in one institution to know what their fellow researchers in other institutions are working on. In addition, some of these repositories are also not updated as frequently as they should be [5].

In a research conducted by Erima, Masai \& Wosyanju [6], with Moi University in Kenya as the case study, it was reported that unless there is a plan for continued access and use of academic research data, there is no guarantee that the research outputs generated today will be available, accessible and useful in the future. This alludes to the need of a national scholarly document repository as well as efficient information retrieval technology to enable retrieval of the consolidated data by interested researchers. The key question this paper addresses is: how can an information retrieval approach be 
developed that ensures relevant research information and meta-data is obtained from research document repositories?

This paper answers the above question and addresses the challenges by making the following key contributions. Firstly, to provide the information retrieval technology required for the retrieval of academic research data from scholarly document repositories, this research, therefore, develops a multilevel text clustering algorithm capable of clustering research outputs (text documents) as well as research metadata in the form of SQL data records. Secondly, in addition to clustering data, this developed approach to text clustering also performs matching and ranking, which are important operations in information retrieval. Thirdly, this research also constructs an information retrieval model, which is composed of the developed algorithm, an identified scholarly document repository [7], as well as a web interface [7] used by researchers to access data from the repository. Finally, we evaluate the effectiveness of the developed text clustering algorithm in the retrieval of academic research data from scholarly document repositories and report very promising results.

The rest of this paper is organized as follows. Section 2 presents related works. Section 3 describes the materials and methods. Section 4 presents the results while Section 5 discusses the results. Finally, Section 6 presents the conclusions and highlights future work.

\section{RELATED WORK}

\subsection{Information retrieval}

The subject of information retrieval, information retrieval systems and information retrieval models has been well covered in literature. Information retrieval is the process of extracting relevant information resources in a predefined and automated manner from an available lot of information resources [8]. In most occasions, this information is usually stored in an information retrieval system. These information retrieval systems make use of information retrieval technology to provide or even suggest documents or information that the application user would find relevant based on their information needs. The information needs of the user are often expressed or represented to the information retrieval systems by the use of a search string or search query. Those documents that the user finds suitable are called relevant documents [9].

An information retrieval system should be in a position to support the following functionalities [9]:

i. The storage and representation of the content or information in the information retrieval system

ii. The representation of a user's information need. In many applications this is usually done via a search query.

iii. The comparison of the two representations (stored information and user's search query). This is usually performed by information retrieval technology or algorithms that search the information retrieval system in order to find information that the user would find relevant.

Information retrieval systems make use of information retrieval models to find suitable information for a particular user. Some of the information retrieval models discussed in literature include the exact match models [9], the vector space model [9] and the latent semantic indexing model [8].

\subsection{Text Clustering in Information Retrieval}

Text clustering is the process of partitioning an unstructured set of objects into groups of similar objects [10]. The goal is usually to have documents in one cluster being as similar as possible, while still being as different from documents in other clusters as possible [10].

Text clustering algorithms are divided into two main groups: hierarchical algorithms (which produce a hierarchy of clusters) and partitioning algorithms (which give a flat partition of the data set) [11]. In addition to these two categories of text clustering algorithms, a distinction is also made between hard and fuzzy clustering. Hard clustering means that a document can only be assigned to one cluster, while on the other hand, fuzzy clustering means that a document can be assigned to more than one clusters [11].

In hierarchical clustering algorithms, clusters are constructed in two main ways: the bottom-up approach and the top-down approach [11]. The bottom up approach is used in agglomerative algorithms. Agglomerative algorithms are deterministic in nature, meaning that they will generate the same cluster hierarchy every time the algorithm is run. On the other hand, the top-down approach is used in divisive algorithms, where any partitioning algorithm are used to split clusters. An example of divisive algorithms is the Bisecting K-Means algorithm [12]. The stopping criteria for both the agglomerative and the divisive algorithms could be the achievement of the required number of clusters, some limit on a criterion function, or any internal evaluation measure.

The K-Means algorithm is probably the most known and most common text clustering algorithm in the category of partitioning text clustering algorithms [13] This algorithm has a time complexity of $O(k n I)$, where $k$ is the number of clusters, $n$ is the number of objects to be clustered while $I$ is the number of iterations that the algorithm runs [14]. Some of the advantages of the K-Means algorithm are that first, it produces tighter clusters than the hierarchical clustering algorithms, and more so if the clusters are globular and second, if variables are huge, the K-Means algorithm most of the times is computationally faster that hierarchical clustering, if $K$ is kept small. One limitation of the K-Means algorithm is that without known information about the data to be clustered, it is difficult to predict a $K$ value (number of clusters to form) that will lead to optimal clustering [11]. Another limitation is that different initial partitions may result in different final clusters.

\subsection{Clustering of SQL data Records}

The integration of data mining algorithms with relational database management systems is both important and challenging at the same time [15]. This has led to the introduction of the concept of clustering among database programmers to aid the process of data mining and analytics. Several research works have explored the use of text clustering algorithms to cluster SQL data records from relational databases. For example, Ordonez [15] explores how the K-Means algorithm can be integrated with a relational database programming application using SQL. In addition, another SQL data clustering algorithm that merges Markov Chain Monte Carlo methods with the EM algorithm is presented by Matusevich and Ordonez [16]. The K-Means algorithm generally performs well, is independent of the operating system used by the programmer and is linear to the 
size of the used dataset [16]. Another algorithm for clustering SQL data is proposed by Sun et al., [17], and is based on depth neural networks and is used to cluster data in distributed databases.

\subsection{Applications of Text Clustering in Information Retrieval}

The role of text clustering in information retrieval cannot be underestimated, as it has a number of applications in this field. These applications differ in the set of texts they cluster (whether it is the search results, the entire collection of text, or a subset of that set of collections) and the aspects of the information retrieval system they try to improves(such as the effectiveness or efficiency of the search system, user interface or user experience) [18]. Some of the applications of text clustering in information retrieval include search result clustering, scatter-gather, collection clustering and clusterbased retrieval [18].

\subsection{Information Retrieval of Academic Research Data}

Information retrieval of academic research data refers to the process of using information retrieval technology to obtain research data from a consolidated store such as a central database or repository. Easy access and retrieval of academic research data has many benefits to researchers. The benefits include easier identification of scientific communities [2], improved efficiency of research and acceleration of innovation.

The increased volume of scholarly data being produced by academic researchers has led to the emergence of the term "Scholarly Big Data" ([2], [19]). Due to this continued growth in the number of publications being produced all over the world as well as other research related data, researchers all over the world get overwhelmed and spend a lot of time when trying to access and retrieve this research data ([2], [20]). In order to help researchers obtain relevant information in this time of information overload, information science specialists need to "develop reliable and effective automated systems that support an easy and effective access to the relevant information" [2]. This demand has necessitated the application of data mining and analysis techniques in the field of scholarly big data, leading to what is now termed as scholarly data mining. In the recent past, various scholars have investigated the application of data mining to solve the problem of access to scholarly data. Some of the main problems attracting attention include general scholarly information access and retrieval ([2], [20], [21]), author disambiguation ([2], [19]), academic recommendation (such as collaborator recommendation as well as literature recommendation) ([2], [19], [22]), expert searching [2], scientific impact evaluation (which could include article or paper impact evaluation, journal impact evaluation, as well as author impact evaluation) ([2], [19], [23]), scholarly data visualization [3] and identification of trending and emerging research topics that are receiving much attention from scholars [22]. Sumba et al., [21] also proposed an architecture using ontologies and data mining to detect similar research areas among researchers as well as potential collaboration networks.

Despite the benefits of having research data being readily available and accessible, availability and accessibility of this data remains a challenge to many scholars in Kenyan universities. ([4], [5], [24]). Needless to say, there is need for an information retrieval model that can cluster data, match the resultant clusters with the user's information needs to identify the relevant clusters, and finally rank the information based on criteria set by the application users (researchers). This will ensure effective information retrieval from scholarly document repositories and enable researchers to realize the aforementioned benefits.

\subsection{Research Gaps}

Existing work did not reveal an available approach for application when the data to be clustered exists both in the form of text documents as well as SQL data records in a relational database. In addition, existing algorithms do not include the matching and ranking operations, hence cannot be used directly when one needs to apply them for information retrieval when issuing search queries. Furthermore, it is clear that accessibility and retrieval of research data is a challenge to the process of carrying out research among Kenyan scholars.

This paper presents a multi-level text clustering algorithm capable of matching and ranking so as to retrieve the most relevant research information and meta-data.

\section{MATERIALS AND METHODS}

\subsection{Research Methods}

\subsubsection{The Research Process}

The research process involved comprehensive literature review, development of the multi-level text clustering algorithm, construction of information retrieval model, and evaluation of the effectiveness of the developed model and associated algorithm as depicted in Figure 1.

The multilevel text clustering algorithm clusters the research metadata (researchers, research projects, funding opportunities among others) in the form of SQL records at the first level, then retrieves and clusters research outputs (research papers, theses, journal articles among others) in the form text documents (PDFs).

The research data information retrieval model is composed of four elements: the developed multilevel text clustering algorithm, an academic research data repository that contains research outputs and metadata, a web interface used by researchers and other system users to access and retrieve information from the repository, and lastly an Application Programming Interface (API) that allows communication between the web interface and the developed algorithm. 


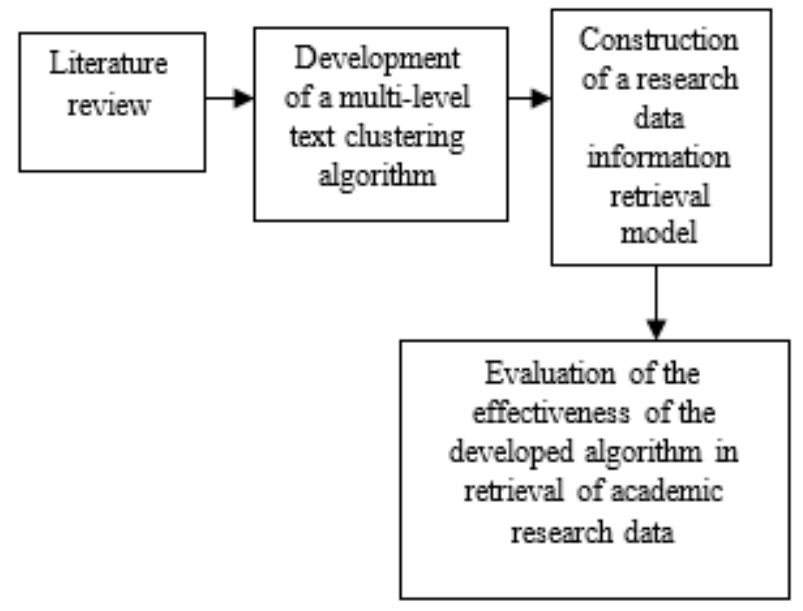

Figure 1. The research process.

The last step in our research process involves evaluating the suitability of the developed multilevel text clustering approach in the retrieval of academic research data from scholarly document repositories.

\subsubsection{Steps Involved in the Development of the Multilevel Text Clustering Algorithm \\ i. $\quad$ Understanding text clustering}

We first set out to study the field of text clustering so as to know the general working and steps of text clustering techniques.

ii. Identification of an existing text clustering technique to use in developing the multilevel approach

After an extensive literature review on text clustering algorithms, we chose to use the K-Means algorithm since it has been well explained in literature and generally performs well in clustering text documents. Since this (K-Means) algorithm was developed to cluster text documents, we tailored it so that it can cluster both SQL records (research metadata) and text documents (research outputs).

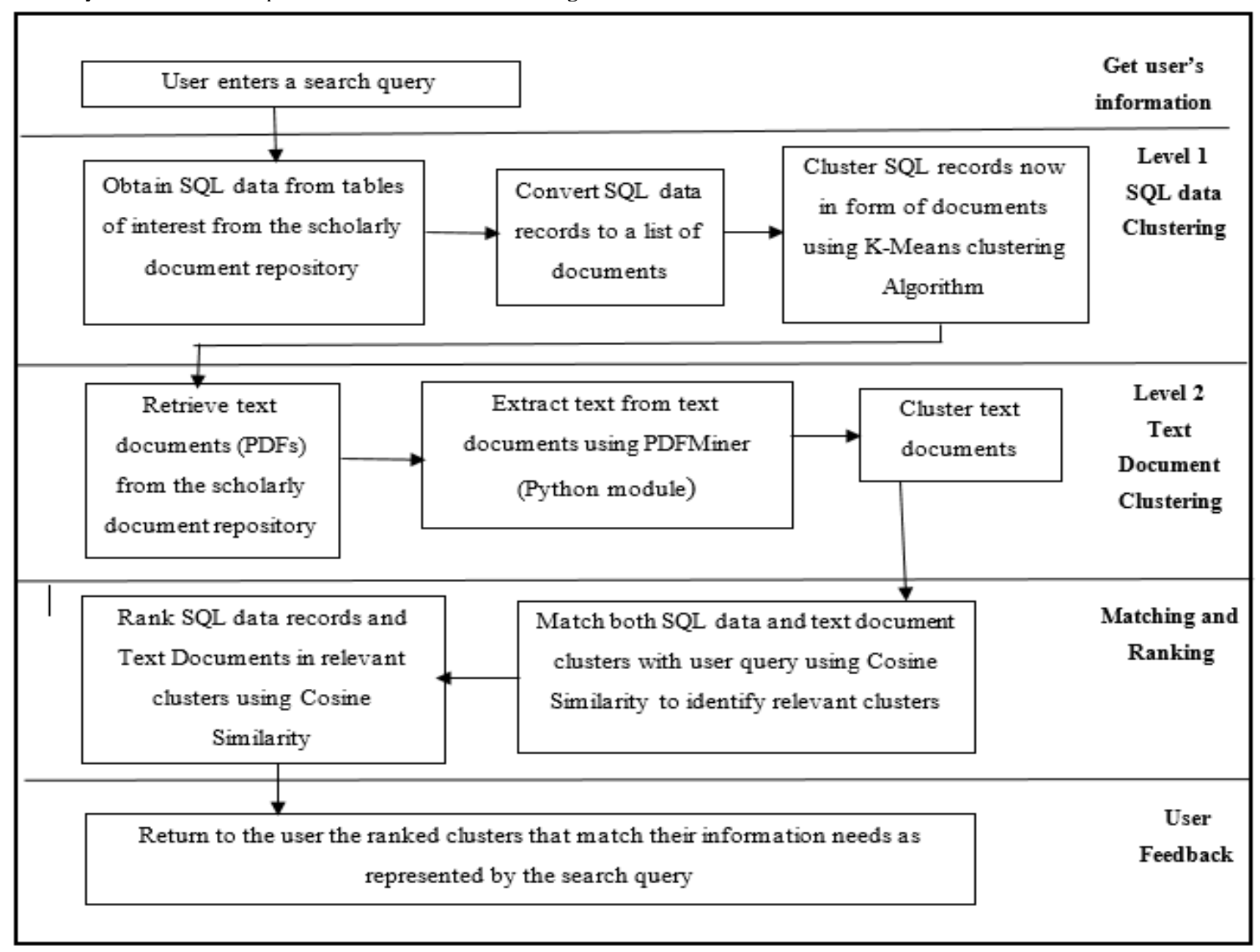

Figure 2. A Graphical Depiction of the Developed Approach to Multi-Level Text Clustering Algorithm

\section{iii. $\quad$ Choosing a similarity measure}

The algorithm has matching and ranking phases that require the use of a similarity measure. The matching phase identifies relevant clusters that are related to a search query from all the clusters in a given category of data. The ranking phase produces relevant information in a ranked order starting with the most relevant when compared to the search query. When various techniques used for similarity measure were reviewed, the Cosine Similarity was chosen to be used in developing the multilevel text clustering approach.

iv. Designing the algorithm 
In designing the algorithm we first came up with the logical steps of the algorithm execution. We also came up with the four phases of the algorithm, as depicted by Figure 2 .

v. Implementation of the multilevel text clustering approach

Once we had made all the decisions regarding the algorithm, we embarked on its implementation. It was developed in such a way that it could cluster both SQL data as well as text documents, as well as perform matching and ranking of information so that the application user would receive ranked information.

\subsubsection{Evaluation of the Developed Multilevel Text Clustering Algorithm}

Once the multilevel text clustering approach was developed, we evaluated its effectiveness in retrieval of research outputs and metadata from scholarly document repositories. The following are the steps that were followed in evaluating the algorithm.

i. Identification of a scholarly document repository Because the developed text clustering approach is meant to cluster both SQL data records as well as text documents, we wanted to work with a repository that has this type of data. We settled on Kenya Research Information System [7] since it has the kind of data we needed.

ii. Choosing the metrics to use in evaluating the algorithm

After carrying out a study on metrics used in evaluating text clustering algorithms, we opted for the Silhouette Coefficient ([25], [12]) and the Davies-Bouldin Index ([25], [12]) metrics. This is because these two metrics are used to measure the internal quality of clusters when the ground truths about the data used for clustering are unknown, which was the case for our data. However, we also evaluated the algorithm on other metrics that are suitable for its application in our application domain, such as its ability to cluster both SQL data and text documents, as well its matching and ranking phases.

iii. Performing Experiments

We performed experiments in a bid to evaluate the effectiveness of the developed text clustering algorithm in the retrieval of research outputs and metadata from scholarly document repositories. We conducted lab experiments in which we used the application to retrieve academic research data like a normal application user would. We did this by supplying a user query as a representation of our information needs. The results of this evaluation are given in section 4 of this paper.

\subsection{System Design}

This section describes the architectural design of the research data information retrieval model, the components of the research data information retrieval model, and the design of the multilevel text clustering algorithm.

\subsubsection{Architectural Design}

The client server architecture was chosen for the research data information retrieval model. This architecture has three components:

$$
i \text {. The client }
$$

In the context of the developed research data information retrieval model, the client refers to a web interface that is used by application users such as researchers when accessing information from the scholarly document repository. The web interface is also used to display search results after information has been retrieved form the repository.

ii. The Server
The server stores the scholarly documents and research metadata in the form of SQL data records in a MySQL database. Research outputs in the form of text documents are stored in the KRIS application file system. The server receives incoming requests from the client, for example request for data in a given research area, then does processing and returns relevant data to the client via a communication network.

\section{iii. Communication Network}

The communication network acts as a link between the client and the server, allowing them to communicate. It allows requests for information from the client to reach the server, as well as retrieved information to be relayed from the server to the client. Figure 3 depicts the architecture.

\subsubsection{Key Components}

The information retrieval model developed in this research has four key components as explained below:

$i$. The multilevel text clustering algorithm This algorithm aids in information retrieval by carrying out clustering of the available research data (research outputs like research papers as well as research metadata in the form of SQL data records). The algorithm clusters both the text documents and the SQL data into clusters of related data. The algorithm also performs matching and ranking so as to return to the user only relevant information based on their information needs as represented by their search query.

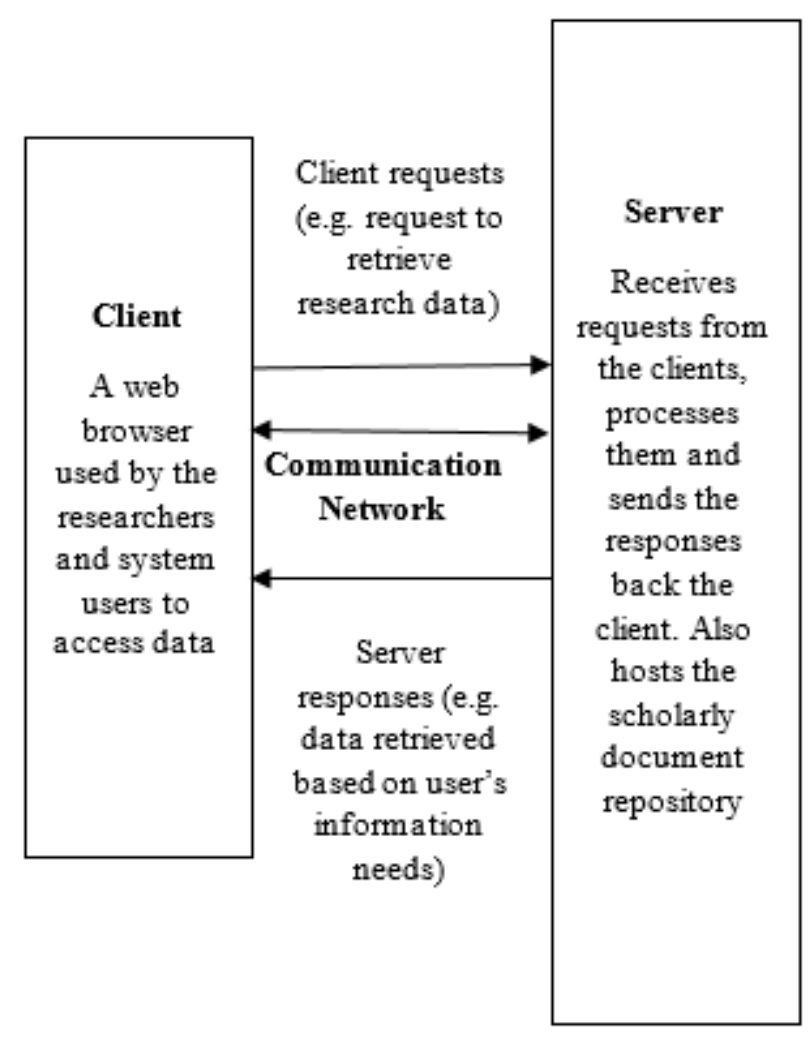

Figure 3. The client server architecture of the developed information retrieval model

\section{ii. A Web interface}

This web interface was developed by Muriithi et al., [7] and is used by researchers and other system users to 
access the data in the scholarly document repository. It has a search text box where the application user provides a search string to represent the kind of information they are interested in retrieving. Search results after information retrieval are also displayed to the user on this web interface.

iii. The scholarly document repository

Research data that is created and used by academic researchers and scholars is consolidated and stored in the repository. The data includes research outputs such as theses, research papers, and journal articles, as well as research metadata such as researchers, research projects, and research project funding opportunities, among others [7]. iv. A Python Application Programming Interface The multilevel clustering approach is developed using Django, which is a Python framework. On the other hand, both the web interface and the scholarly document repository are developed using Laravel, a PHP framework. Due to having different applications in different languages and frameworks, it became necessary to create an API to allow the two applications to communicate.

Figure 4 shows how these four components are layered in the academic data information retrieval model. On the other hand, Figure 5 shows the relationship between the Laravel framework application, the Django framework application and the API.

\section{Academic Research Data Information Retrieval Model}

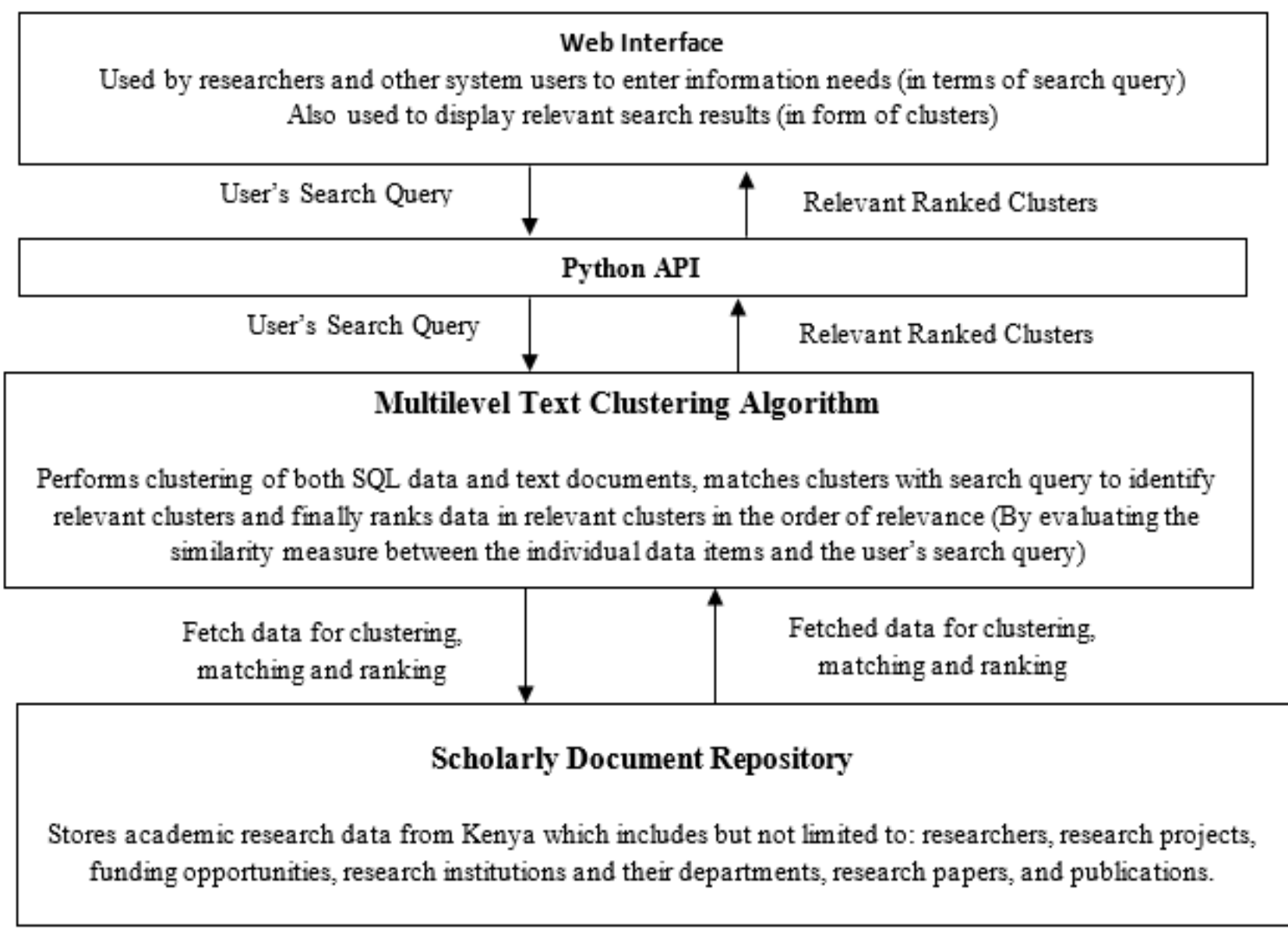

Figure 4. Components of the Constructed Academic Research Data Information Retrieval Model.

\subsubsection{The design of the multilevel text clustering algorithm}

The algorithm presented in this paper clusters both SQL data records in a database as well as text documents in the form of PDFs. The algorithm also performs matching and ranking of clusters in relation to their degree of similarity with the user query. In designing the algorithm, we came up with four logical steps in the execution of the algorithm. Figure 2 depicts these four logical steps described below:

www.ijcat.com i. Retrieving data from the scholarly document repository

This step gets data from the repository so that it can be clustered. This data includes research metadata such as researchers and research projects, in the form of SQL records in a MySQL database, as well as research outputs such as research papers in the form of PDFs.

ii. SQL Data clustering 
This steps leads to the grouping of similar data in same clusters. The algorithm clusters research metadata in the form of SQL data records in the first level, and then clusters research outputs (text documents like research papers) in the second level.

\section{iii. $\quad$ Matching}

During this step, all the clusters in a given category of data are compared with the search query supplied by the application user in order to identify the relevant clusters.

iv. $\quad$ Ranking

This is the last phase of the algorithm. It takes as input the search query and the relevant cluster elements identified in the matching phase. It then calculates the similarity measure of each element in the relevant clusters when compared to the search query using Cosine Similarity. The output of this step is a ranked list of relevant data in each category of data that is the given back to the application user as search results.

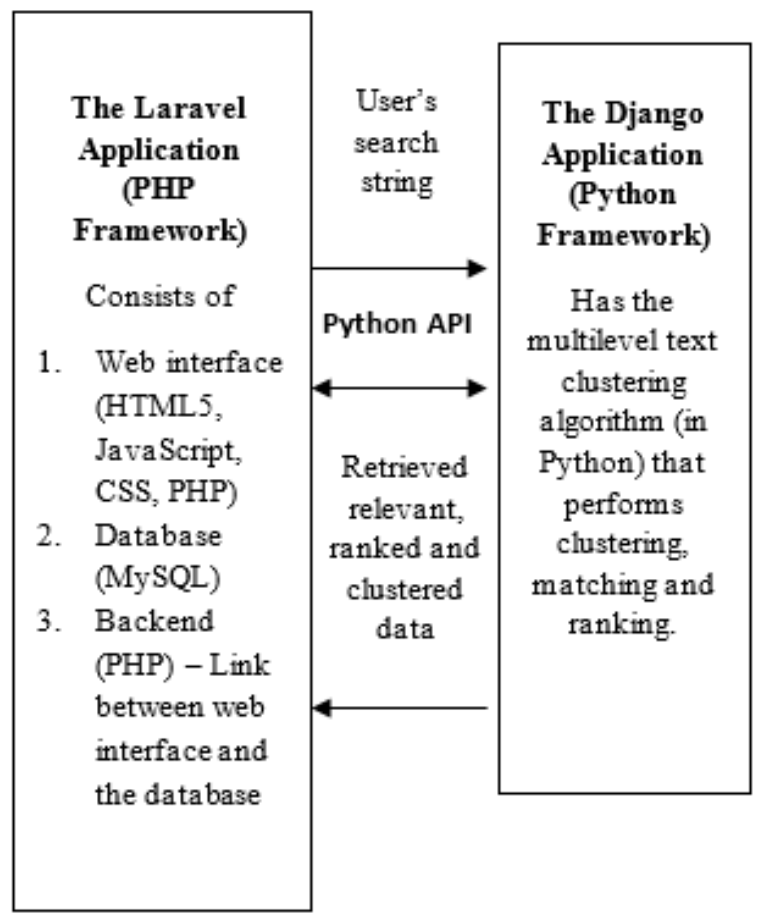

Figure 5. The relationship between the Python API, the Laravel application and the Django application.

\subsection{Implementation of the Multi-level Text Clustering Algorithm}

To implement the multi-level text clustering approach, we used Python, the Django framework, and the Pycharm Integrated Development Environment (IDE).

\subsubsection{Clustering of Text Documents (Research Outputs) \\ i. $\quad$ Extraction of Text From PDF Documents}

As noted earlier, the scholarly document repository contains, in PDF format, research outputs like postgraduate theses, research papers, etc. To cluster the documents, first, we used PDFMiner, a Python library, to extract the text from the documents. PDFMiner is able to extract the text such that all the text from one document forms one long string of characters. These strings of characters are then stored in a Python list, which then becomes the corpus that the algorithm clusters.

ii. $\quad$ Clustering

www.ijcat.com
As earlier mentioned, K-Means algorithm was used in text clustering. We installed scikit-learn, an open source Python machine learning library that has a number of data mining and data analysis tools, in order to implement K-Means.

iii. Feature Extraction Using TF-IDF

Since the K-Means algorithm only works with numbers, we had to do feature extraction so as to obtain numbers from the document corpus. In our multilevel text clustering approach, feature extraction was performed using a scikit-learn tool called TfidfVectorizer. TfidfVectorizer uses an in-built Python dictionary to map the words in a document to feature indices and thereafter compute a word frequency matrix. The resultant word frequencies are then reweighted using the Inverse Document Frequency (IDF) vector collected featurewise over the document corpus.

iv. Pseudo Code for the Clustering Step

\author{
Procedure: Text Clustering Algorithm \\ Input: A list of text documents \\ Output: Text document clusters \\ Begin \\ Convert text documents list to a vector space model \\ Cluster text documents using K-means \\ Return text document clusters \\ End
}

Figure 6. Algorithm for clustering text documents (research papers)

The algorithm shown in Figure 6 shows the steps involved in the clustering of text documents.

\subsubsection{Clustering of SQL Data Records (Research Metadata)}

The research metadata in the scholarly document repository is stored in a MySQL database in the form of SQL data records. To be able to cluster this data, first we fetch it from the database and convert records to a list of strings. Each record in the form of string of characters becomes a document in the list. This document list is then clustered using K-Means algorithm, and the resultant clusters assigned to a global variable so that they can be available for both the matching and ranking phases of the algorithm. Figure 7 shows the algorithm for the important steps of clustering SQL data. In this example we are clustering data in the 'researchers' table.

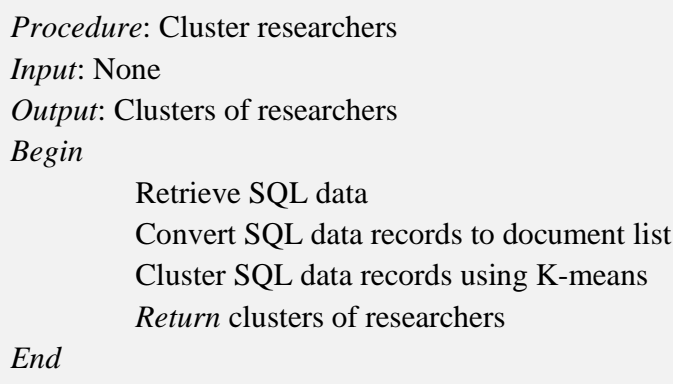

Figure 7. Algorithm for clustering of SQL data 


\subsubsection{The Matching Phase of the Algorithm}

The importance of the matching phase of the developed multilevel text clustering algorithm is to identify relevant clusters from all the cluster formed. All the clusters formed are compared to the search query using Cosine Similarity, and those found to be similar are identified as candidates whose elements will be used in the ranking phase.

Before matching, the user's search string has its stop words removed. We wrote a Python function for that purpose. The function receives the search string as entered by the application user as an argument, and returns it without stop words. To achieve this, we calculated the set difference between the Scikit-learn's frozen set of English stop words and the set of words in the user's search query.

The algorithm in Figure 8 shows the step by step process of the matching phase, clearly indicating the inputs and outputs of that process. In this example we were matching researchers' clusters with the user's search query in order to get the clusters containing relevant researchers.

Procedure: Match researchers clusters

Input:User's search query; Researchers clusters

Output: Relevant researchers clusters

Begin

Retrieve the search string minus stop words

Match researchers clusters with user's search query (without stop words) using Cosine Similarity

Return relevant researchers clusters (those with similarity measure greater than zero (0))

End

Figure 8. Algorithm for matching clusters to user query

\subsubsection{The Ranking Phase of the Algorithm}

In this phase, the documents or SQL data records in the relevant clusters identified in the matching phase are arranged in the order of their similarity when compared to the user's information needs as expressed by their search query, starting with the most similar. Just like in the matching phase, we also use the Cosine Similarity to measure the similarity between the search query (without its stop words) and the elements in the relevant clusters. In addition to creating a ranked list of relevant documents and data records, the ranking phase helps to remove documents or data records that mistakenly end up in the identified relevant clusters (outliers). This is because documents with a similarity measure of zero (0) (meaning that they are completely dissimilar to the search query) when compared to the search query are not included in the list of relevant and ranked documents and data records to be returned to the application user. The pseudo code for this ranking phase is shown in Figure 9.

Procedure: Rank relevant researchers

Input: Relevant researchers clusters; User's search string

Output: Ranked relevant researchers

Begin

Retrieve the search string minus stop words

Rank relevant researchers according to their

degree of relevance based on the user's search string using Cosine Similarity

Return ranked relevant researchers
Figure 9. Algorithm for ranking cluster results

\section{RESULTS}

The aim of this section is to evaluate the effectiveness of the developed multilevel text clustering algorithm in the retrieval of academic research data, both in the form of SQL data records and also text documents in PDF format. The data used for evaluation was consolidated research data from a central research data repository [7]. This data is composed of research papers (text documents) from academic researchers as well as research metadata stored in a MySQL database. The tables used for evaluation from this database keep data about researchers, research projects, and funding opportunities that researchers can apply for to facilitate their research. More details about this scholarly document repository can be found in [7].

\subsection{Results of Evaluating the Algorithm's Ability to Cluster Both SQL Data Records and Text Documents}

The aim of developing the algorithm was so that it can be able to cluster both SQL data and text documents from scholarly document repositories. We evaluated the algorithm against this criteria and it was indeed able to cluster both SQL data and research papers (text documents).

With regard to SQL data clustering, we clustered data in three tables: the researchers' table, the research projects table and the funding opportunities table. We chose to cluster data in both the researchers' and research projects tables into seven (7) clusters while data in the funding opportunities table was clustered into five clusters. The choice of number of resultant clusters was based on prior knowledge of the data that was being clustered.

In the graphs shown in Figure 10, Figure 11, Figure 12 and Figure 13, the $\mathrm{X}$ and $\mathrm{Y}$ coordinates represent the principal components for the two artificial dimensions created by the Principal Component Analysis (PCA) algorithm after dimensionality reduction.

The researchers' table stores bio data about researchers such as their research institution, department, titles (Prof., Dr., etc) and research interests. Figure 10 shows the 7 clusters resulting from clustering the data in this table. The cluster centroids for these clusters are indicated using digits (1-7). These digits also serve as the cluster labels.

The common characteristic of all the researchers in one cluster was the research area of interest. For example, cluster 7 was composed of researchers who had interests in banking, all of them belonging to either Jomo Kenyatta University of Agriculture and Technology or the University of Nairobi. In addition, cluster 4 had researchers belonging to various departments and research institutions, but having text clustering as their research interest. The cluster labeled "3" was made up of researchers specializing in HIV and AIDS, but from various research institutions and departments. In addition, the cluster labelled " 6 " had researchers with interests in accounting and finance, from different universities and departments. Cluster 2 was composed of clusters of researchers who were cancer specialists. The cluster labeled 
"1" was made up of food security specialists from The University of Nairobi. Last but not least, the fifth cluster had researchers with research interests in cloud computing. All the researchers in that cluster (5) came from either Kenyatta University, the University of Nairobi or Jomo Kenyatta University of Agriculture and Technology.

The research projects table has data relating to research projects carried out by scholars such as the projects' title and project abstract. Figure 11 represents the seven clusters resulting from clustering data in this SQL table. Of these 7 clusters, five of them can be clearly seen. These five clusters represent research projects in the research areas of cancer (cluster 1), food security (cluster 2), HIV and AIDS (cluster 3 ), cloud computing (cluster 5) and finally text clustering (cluster 6). However, there are two clusters (clusters 4 and 7) that appear very close together. These clusters belong to projects in the banking (cluster 4 ) and accounting and finance (cluster 7) sectors, and they have very similar terms. That is why the cluster centroids for the data in these two clusters appear very close. The seven clusters from the research projects table shown in Figure 11 represents projects in seven different research areas of data used in this research.

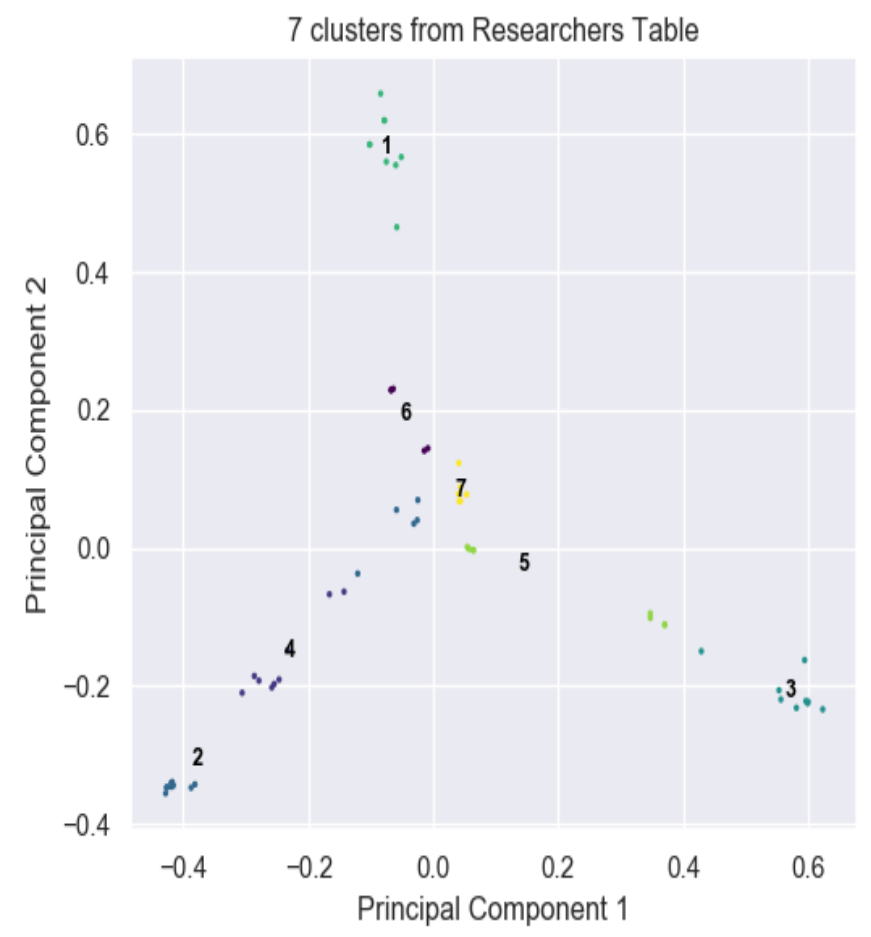

Figure 10. Results of clustering SQL data in the researchers table.

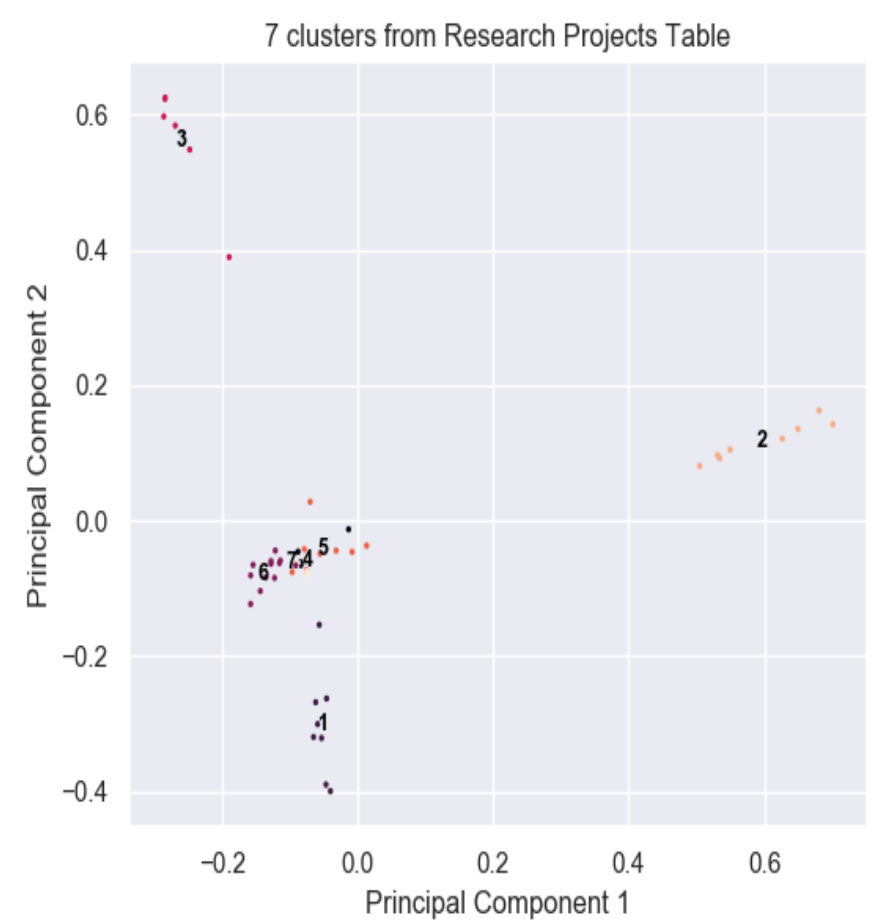

Figure 11. Clusters resulting from clustering data in the research projects table

In the funding opportunities table, the data stored include the funding body, the research areas being funded, and the deadline for applying for the funding opportunity. The five (5) clusters resulting from clustering these SQL data records are represented in Figure 12 with the cluster centroids shown by digits (1-5). Each of these five clusters contains all the research opportunities for projects in various research areas provided by one funding body. So the unique attribute in these five clusters is the name of the funding organization or body.

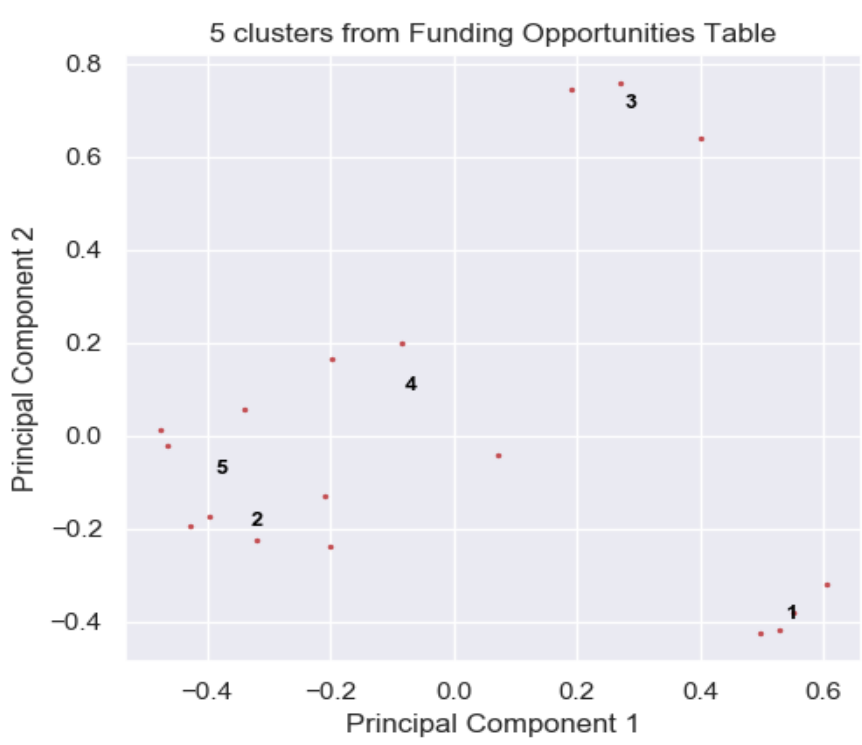

Figure 12. The 5 clusters formed after clustering data in the Funding opportunities table. 


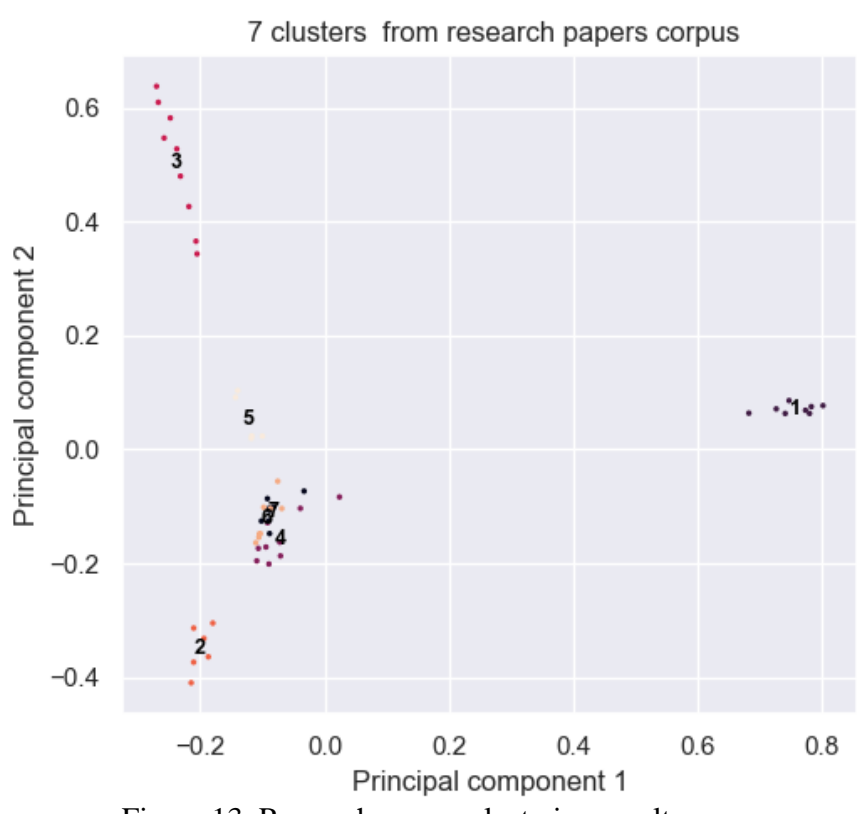

Figure 13. Research papers clustering results

To evaluate the ability of the developed multilevel text clustering approach to cluster text documents, we used research papers with content from seven research areas. These research areas include: text clustering, cancer, HIV and AIDS, banking, accounting and finance, food security and cloud computing. Because of this, we chose seven (7) to be the number of clusters to be generated by the algorithm. The algorithm generates seven clusters, grouping all the research papers in one research area in one cluster. Clusters 6 and 7 appear very close together because they represent research papers with content from two similar fields, hence making them to have similar terms. These two fields are banking and accounting and finance respectively. The 7 resulting clusters are depicted in Figure 13.

\subsection{Results of Evaluating the Quality of Clustering Using the Silhouette Coefficient Metric}

The Silhouette Coefficient is an internal quality measure of clustering results, and it is used when the ground truth labels of data being clustered are not known ([25], [12]) (which is the case for the data used to evaluate the developed algorithm). The Silhouette score indicates how similar a point is to the cluster it has been assigned. The Silhouette values are bounded between -1 and 1 . The result is 1 for perfectly formed clusters and -1 for poorly formed clusters.

Table 1 shows the Silhouette Coefficient scores for the researchers' clusters shown in Figure 10, the research projects clusters represented in Figure 11, the funding opportunities clusters shown in Figure 12, and lastly the research papers clusters illustrated in Figure 13. According to these scores, which is something that can also be noticed from the graphical representation of the clusters, data in the researchers table forms the best quality of clusters.
Table 1. Silhouette Coefficient scores for the four categories of clusters formed

\begin{tabular}{|l|l|l|}
\hline S.NO & Categories of Clusters & $\begin{array}{l}\text { Silhouette Coefficient } \\
\text { scores }\end{array}$ \\
\hline 1 & Researchers & 0.2137977853179148 \\
\hline 2 & Research projects & 0.10267977158553505 \\
\hline 3 & Funding opportunities & 0.1976613904708869 \\
\hline 4 & $\begin{array}{l}\text { Text documents } \\
\text { (research papers) }\end{array}$ & 0.20310426032008072 \\
\hline
\end{tabular}

\subsection{Results of Evaluating the Quality of Clustering using the Davies-Bouldin Index}

Table 2. The Davies-Bouldin Index scores for the researchers, research projects, funding opportunities and research papers clusters

\begin{tabular}{|l|l|l|}
\hline S.NO & Categories of Clusters & $\begin{array}{l}\text { Davies-Bouldin Index } \\
\text { scores }\end{array}$ \\
\hline 1 & Researchers & 1.3715487874474703 \\
\hline 2 & Research projects & 1.9340321303767844 \\
\hline 3 & Funding opportunities & 1.445894695752895 \\
\hline 4 & $\begin{array}{l}\text { Text documents (research } \\
\text { papers) }\end{array}$ & 1.4357250322273192 \\
\hline
\end{tabular}

Just like the Silhouette Coefficient, the Davies-Bouldin Index is an internal quality measure, i.e., it uses the clusters themselves and not any other known external information such as labels ([25], [12]). The evaluation metric returns the ratio between the intra cluster distances and inter cluster distances. The lowest value is zero, and the lower the scores, the better the clustering. The Davies-Bouldin Index scores for the formed clusters are shown by Table 2 . According to these results, clusters resulting from the researchers table resulted in the best formed clusters, since they have the least DaviesBouldin Index score. This is in tandem with the results obtained from using the Silhouette Coefficient metric. 


\subsection{Results of Evaluating the Matching Phase of the Algorithm}

Table 3. Relevant funding opportunities clusters identified at the matching phase of the algorithm

\begin{tabular}{|c|l|l|}
\hline $\begin{array}{c}\text { Cluster } \\
\text { Number }\end{array}$ & Funder Name & $\begin{array}{l}\text { Research Area } \\
\text { being Funded }\end{array}$ \\
\hline \multirow{5}{*}{1} & Africa ai Japan & $\begin{array}{l}\text { Accounting, } \\
\text { Finance }\end{array}$ \\
\cline { 2 - 3 } & Africa ai Japan & Text Clustering \\
\cline { 2 - 3 } & East Africa Research Fund & Text Clustering \\
\cline { 2 - 3 } & Africa ai Japan & Food Security \\
\hline \multirow{5}{*}{2} & Kenya Research Fund & $\begin{array}{l}\text { Accounting, } \\
\text { Finance }\end{array}$ \\
\cline { 2 - 3 } & Kenya Research Fund & Text Clustering \\
\cline { 2 - 3 } & Kenya Research Fund & $\begin{array}{l}\text { Cloud } \\
\text { Computing }\end{array}$ \\
\cline { 2 - 3 } & Kenya Research Fund & Cancer \\
\hline \multirow{5}{*}{3} & $\begin{array}{l}\text { National Commission for Science, } \\
\text { Technology and Innovation }\end{array}$ & HIV and AIDS \\
\cline { 2 - 3 } & $\begin{array}{l}\text { National Commission for Science, } \\
\text { Technology and Innovation }\end{array}$ & $\begin{array}{l}\text { Accounting, } \\
\text { Finance }\end{array}$ \\
\cline { 2 - 3 } & $\begin{array}{l}\text { National Commission for Science, } \\
\text { Technology and Innovation }\end{array}$ & Text Clustering \\
\cline { 2 - 3 } & & \\
\hline
\end{tabular}

The importance of this evaluation is to find out if the algorithm is able to identify all the clusters that contain elements similar to the search string provided by the application user in KRIS. The elements in these relevant clusters are candidates for ranking in the ranking phase of the algorithm.

In this particular run of the algorithm, the search string (user query) provided was "text clustering". In the matching phase of the algorithm, from the five (5) funding opportunities clusters shown in Figure 12, the matching phase identified three (3) relevant clusters that contain "text clustering". These identified funding opportunities clusters are shown by Table 3.

\subsection{Results of Evaluating the Ranking Phase of the Algorithm}

The ranking phase of the algorithm uses Cosine Similarity measure to compare all the elements in the relevant clusters identified in the matching phase with the user's search string. These elements are then arranged in a list starting with the most similar. This ranked list of relevant data is then returned to the user via the KRIS web interface.

Table 4 shows the Cosine Similarity measures for all the relevant funding opportunities present in the relevant clusters shown in Table 3. On the other hand, Table 5 shows the ranked relevant funding opportunities based on the user's query ("text clustering").

Table 4. Cosine Similarity measures of funding opportunities in identified relevant clusters

\begin{tabular}{|c|l|l|l|}
\hline $\begin{array}{l}\text { Cluster } \\
\text { Number }\end{array}$ & Funder Name & $\begin{array}{l}\text { Research Area being } \\
\text { Funded }\end{array}$ & $\begin{array}{l}\text { Cosine } \\
\text { Measure }\end{array}$ \\
\hline \multirow{5}{*}{} & Africa ai Japan & Accounting, Finance & 0.0 \\
\cline { 2 - 4 } & Africa ai Japan & Text Clustering & 0.566345204707 \\
\cline { 2 - 4 } 1 & East Africa Research Fund & Text Clustering & 0.437223120979 \\
\cline { 2 - 4 } & Africa ai Japan & Food Security & 0.0 \\
\hline \multirow{3}{*}{2} & Kenya Research Fund & Accounting, Finance & 0.0 \\
\cline { 2 - 4 } & Kenya Research Fund & Text Clustering & 0.61920901471 \\
\cline { 2 - 4 } & Kenya Research Fund & Cloud Computing & 0.0 \\
\cline { 2 - 4 } 3 & Kenya Research Fund & Cancer & 0.0 \\
\cline { 2 - 4 } & National Commission for Science, Technology and Innovation & HIV and AIDS & 0.0 \\
\cline { 2 - 4 } & National Commission for Science, Technology and Innovation & Accounting, Finance & 0.0 \\
\cline { 2 - 4 } & National Commission for Science, Technology and Innovation & Text Clustering & 0.457674795248 \\
\hline
\end{tabular}

Table 5. Relevant and ranked funding opportunities after searching for "text clustering"

\begin{tabular}{|c|l|l|l|}
\hline $\begin{array}{l}\text { Cluster } \\
\text { Number }\end{array}$ & Funder Name & Research Area being Funded & Cosine Similarity Measure \\
\hline 2 & Kenya Research Fund' & Text Clustering & 0.61920901471 \\
\hline 1 & Africa ai Japan & Text Clustering & 0.566345204707 \\
\hline 3 & $\begin{array}{l}\text { National Commission for Science, Technology } \\
\text { and Innovation }\end{array}$ & Text Clustering & 0.457674795248 \\
\hline 1 & East Africa Research Fund & Text Clustering & 0.437223120979 \\
\hline
\end{tabular}

For data to be added to the list of what is to be returned to the user, the similarity measure has to be greater than zero. So from Table 4, only the four funding opportunities shown in
Table 5 are returned to the user as relevant information. The data is ranked, starting with the most relevant. 


\subsection{Results of Using the Algorithm to Retrieve Data from KRIS via a web interface}

Since the algorithm is invoked from KRIS through a Python API, this evaluation is to find out whether the algorithm indeed is able to return ranked and relevant data to the application user. Figures 14-16 show the results returned by the multilevel text clustering algorithm after searching for the word "cancer". Specifically, Figure 15 shows the relevant

research projects, and Figure 16 contains a list of all relevant research papers. For the research papers, only the title of the paper is displayed. All these data have been ranked so that the most relevant data is on the top of the list. Figure 14 is basically the KRIS search results interface. It indicates when the search is complete, the number of relevant items retrieved, the user's search string, and the amount of time it took to retrieve the information from the database and file system (for research papers) and display it in the search results interface. The time is given in milliseconds.

In the event that what the KRIS application user has provided as a search query does not match any of the SQL data stored in the database or any of the research outputs stored in the applications file system, the application notifies the user that no relevant information was found for retrieval and requests them to redefine the search query and try again.

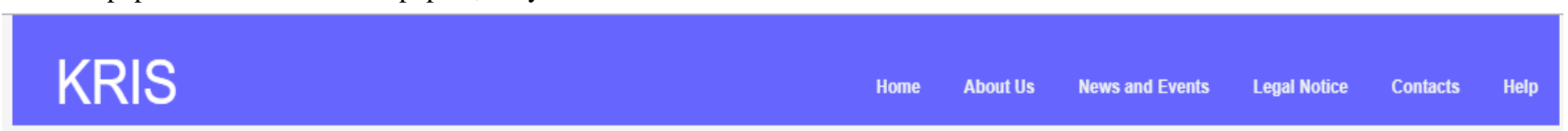

\section{KENYA RESEARCH INFORMATION SYSTEM}

Q Searching Search complete

Showing results for "Cancer"

Search results 17 results found

Funding opportunities:

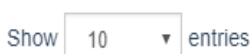

Search:

Figure 14. KRIS application search results interface 
International Journal of Computer Applications Technology and Research

Volume 8-Issue 03, 66-81, 2019, ISSN:-2319-8656

\begin{tabular}{|c|c|c|c|c|c|c|}
\hline \multicolumn{2}{|c|}{ KRIS } & Home & \multicolumn{2}{|c|}{ News and Events } & \multirow[t]{2}{*}{ Contacts } & login/Register \\
\hline \multicolumn{6}{|c|}{ Research Projects: } & \\
\hline S/NO & Project Title & Researcher & $\begin{array}{l}\text { Project } \\
\text { Research } \\
\text { Areas }\end{array}$ & Research Institution & Department & Action \\
\hline 1 & $\begin{array}{l}\text { FACTORS ASSOCIATED WITH BREAST CANCER AMONG WOMEN } \\
\text { PATIENTS ATTENDING KENYATTA NATIONAL HOSPITAL, KENYA - } 2008\end{array}$ & $\begin{array}{l}\text { Dr. REUBEN } \\
\text { SHIKANGA }\end{array}$ & Cancer & $\begin{array}{l}\text { Jomo Kenyatta University of } \\
\text { Agriculture and Technology }\end{array}$ & $\begin{array}{l}\text { Community Health } \\
\text { and Development }\end{array}$ & View \\
\hline 2 & CANCER: A MOLECULAR CURSE? & $\begin{array}{l}\text { Dr. NGUGI M. } \\
\text { PIERO }\end{array}$ & Cancer & The University of Nairobi & $\begin{array}{l}\text { Biochemistry and } \\
\text { Biotechnology }\end{array}$ & View \\
\hline 3 & $\begin{array}{l}\text { ANTIOXIDANT AND ANTIPROLIFERATIVE ACTIVITIES OF PLANT DERIVED } \\
\text { EXTRACTS AGAINST CERVICAL AND PROSTATE CANCER CELL LINES. }\end{array}$ & $\begin{array}{l}\text { MrS. KEDESANI } \\
\text { DOROTHY }\end{array}$ & Cancer & $\begin{array}{l}\text { Jomo Kenyatta University of } \\
\text { Agriculture and Technology }\end{array}$ & $\begin{array}{l}\text { Community Health } \\
\text { and Development }\end{array}$ & View \\
\hline 4 & $\begin{array}{l}\text { CERVICAL CANCER SCREENING UPTAKE AMONG WOMEN ATTENDING } \\
\text { NAIVASHA COUNTY REFERRAL HOSPITAL }\end{array}$ & $\begin{array}{l}\text { Mrs. SERAH } \\
\text { FAITH WANJIRU } \\
\text { MBATIA }\end{array}$ & Cancer & $\begin{array}{l}\text { Jomo Kenyatta University of } \\
\text { Agriculture and Technology }\end{array}$ & $\begin{array}{l}\text { Community Health } \\
\text { and Development }\end{array}$ & View \\
\hline 5 & $\begin{array}{l}\text { Cancer Genetics Education in a Low- to Middle-Income Country: Evaluation of } \\
\text { an Interactive Workshop for Clinicians in Kenya }\end{array}$ & Dr. Helen Dimaras & Cancer & The University of Nairobi & Human Pathology & View \\
\hline
\end{tabular}

Figure 15. Relevant and ranked research projects information retrieved from the database after searching for "cancer"

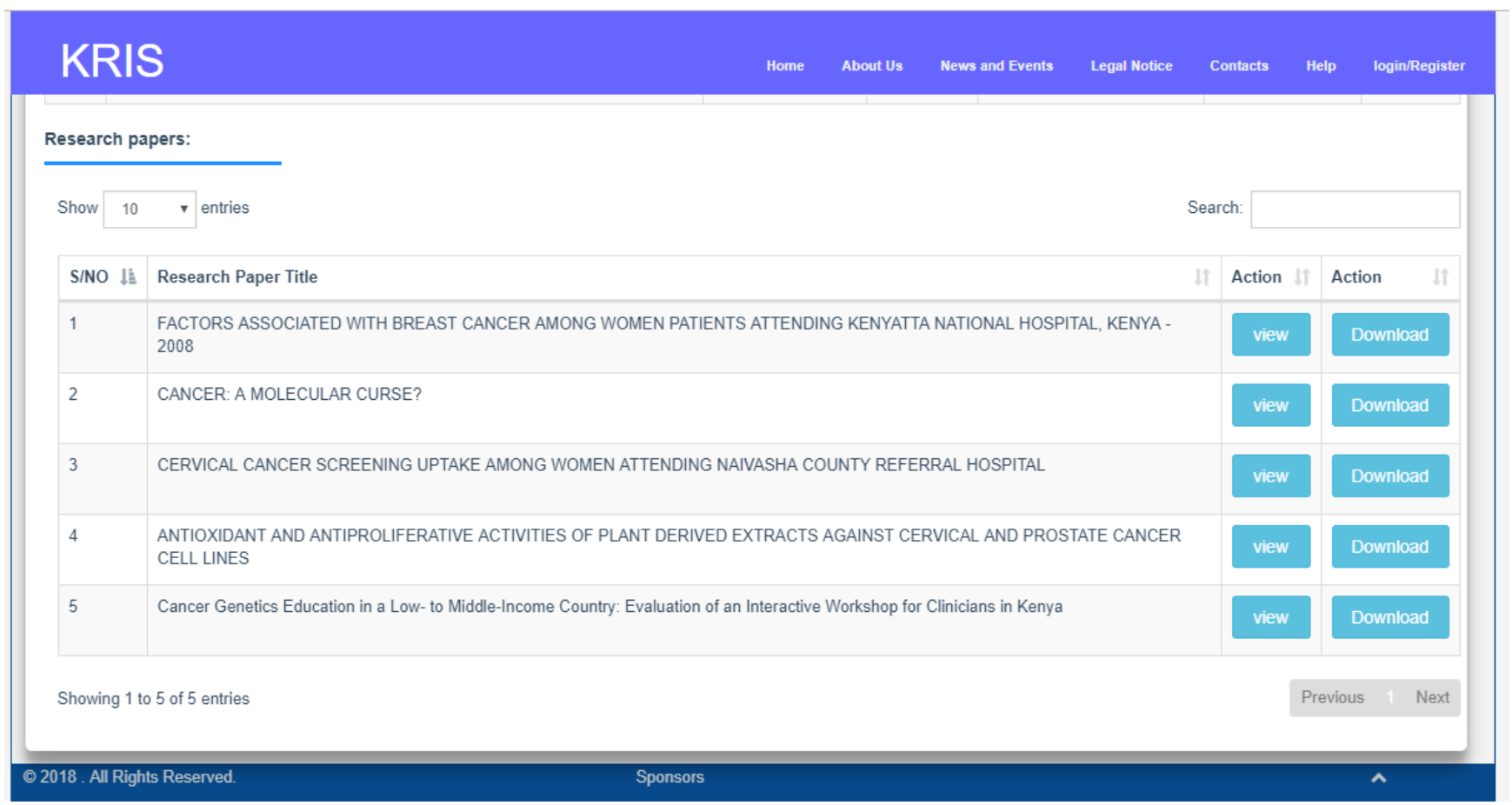

Figure 16. Relevant and ranked research papers retrieved from the file system of KRIS application after searching for "cancer"

\section{DISCUSSION}

From the evaluation results, it is clear that the developed multilevel text clustering algorithm meets its objective: to be able to cluster both SQL data and text documents, making it applicable in the retrieval of research outputs and metadata from scholarly document repositories.

Results from evaluating the algorithm on its ability to cluster both SQL data and text documents show that the algorithm is indeed able to fetch data from an SQL database and cluster it, as well as extract the content of text documents (research papers) in PDF format and cluster them. The resultant clusters

www.ijcat.com have related data being grouped together, achieving the goal of clustering.

The Silhouette Coefficient scores are bounded between -1 and 1. A score of one means perfect clusters while a score of -1 , which is the lowest possible, means that the clusters are very poorly formed. A score of zero (0) means that the cluster elements are at the border of other clusters. Generally, negative values indicate bad clustering while positive values indicate good clustering. On the positive side, the larger the value the better the clustering. According to Table 1, all the scores for the four categories of clusters are positive values, 
indicating relatively good clustering. However, these values are still far from 1 (which is the perfect score). This can be attributed to the values of $K$ (number of clusters to form per group of data) chosen when initializing the clustering algorithm. The values of $K$ chosen to initialize the algorithm were just based on the researchers known information of the data to be clustered. For example, for the research papers, a value of seven (7) was used as the number of clusters to form $(K)$ since in total there were research papers from seven research areas.

According to Table 1, the research projects clusters, with the least score $(0.10267977158553505)$ were the worst formed compared to the other three categories of clusters, while the researchers clusters were the best formed with a Silhouette Coefficient of 0.2137977853179148 . Possibly, choosing an optimal number of clusters would lead to better clustering and higher values for the Silhouette Coefficient metric.

The Davies-Bouldin Index scores shown in Table 2 do validate the evaluation results given by the Silhouette Coefficient metric in Table 1. In Table 2, the research projects clustering are the worst formed since they have the largest score, which is what the Silhouette Coefficients in Table 1 also imply. The researchers clusters are still the best formed according to the Davies-Bouldin Index scores, with a score of 1.3715487874474703 . The choice of the number of clusters to form at the end of the clustering process as well as the choice of the maximum iterations when initializing the algorithm are the reason why the Davies-Bouldin Index scores are not zero (to imply perfect clustering).

Results from evaluating the algorithm on its ability to match resulting clusters with the user's search query in order to identify matching clusters show correct working of the matching phase of the algorithm. Table 3 shows the three clusters (out of the total five funding opportunities clusters) that were found to have information matching the user's search string (text clustering). In those clusters, the first cluster was found to be relevant because it contained two funding opportunities, one by the East Africa Research Fund and the other one by Africa ai Japan for text clustering research projects. Likewise, the second cluster has information about the Kenya Research Fund providing funding for text clustering projects. Lastly, the third cluster has the last record indicating that the National Commission for Science, Technology and Innovation is also providing a funding opportunity for research projects dealing with text clustering. In all the five funding opportunities clusters produced by the algorithm, only the clusters in Table 3 matched the user's information needs (text clustering).

Table 4 has all the funding opportunities in the three funding opportunities clusters in Table 3 identified in the matching phase to be relevant. These funding opportunities (in Table 4) also have a similarity measure score, indicating the extent to which they are similar to the user's search string (text clustering). In Cosine Similarity, the scores are bounded between zero ( 0 ) and one (1). A score of 0 means no similarity at all while a score of 1 means a perfect match. This implies that the larger the score, the more similar two strings are. Based on the similarity scores in Table 4, only four records have a degree of similarity with the search string, since the rest have a Cosine Similarity measure of 0.0 , meaning no similarity at all. Table 5 then shows those four matching funding opportunities ranked in a single list, starting with the one that is most similar (and therefore most relevant when it comes to information retrieval). The first one provided by the Kenya Research Fund has a score of
0.61920901471, while the last one by East Africa Research Fund has the least score of 0.437223120979 . Obviously, from Table 4 and Table 5, and the explanation given so far about the results of the ranking phase of the algorithm, the ranking phase of the developed algorithm is working as it should, ensuring that the most relevant information is kept on top of the information returned by the algorithm to the calling program (KRIS).

The evaluation of the algorithm on its ability to enable the user to retrieve data from a scholarly document repository tested all the steps of the processing of the algorithm illustrated in Figure 2. It tests whether the user's information needs in the form of a user query or search string can be send to the algorithm via the API. It also tests the ability of the algorithm to retrieve SQL data from the KRIS database and as well as research papers from the application's file system, including clustering, matching and ranking the relevant data according to its degree of similarity with the user's information needs. In addition, this evaluation also tests the ability of the algorithm to send back the relevant and ranked information retrieved from the database and the relevant text documents (research papers) to the KRIS search results interface. Figure 15 and Figure 16 do show that the algorithm does allow a researcher or any other application user to retrieve academic research data from KRIS via a web interface. For the data from the database (funding opportunities, researchers and research projects), the application user can click on the "view" button in the data tables to have access to more information about a given record that is not displayed in the data tables. In as far as the returned research papers are concerned, the researcher can opt to just view them to read the text or download the research papers to read them later by using the respective button.

\section{CONCLUSION AND FUTURE WORK}

This paper addresses the problem of access and retrieval of research information among researchers in Kenyan institutions of higher learning as well as research institutes. It develops a multilevel text clustering algorithm to be applied in the clustering and retrieval of academic research data from scholarly document repositories. The algorithm is able to fetch and cluster SQL data (research metadata such as researchers' biodata) at the first level, and extract text from text documents (research outputs such as research papers in the form of PDFs) and cluster them in the second level. In addition to text clustering, the developed approach to text clustering also performs matching and ranking, both of which are important operations in information retrieval. In addition, this paper also develops a research data information retrieval model by integrating the algorithm with a research data repository to facilitate retrieval of academic research data from the repository through a web interface. This information retrieval model is applicable not only for the retrieval of scholarly data, but also in any other situation where information to be retrieved consists of text documents and SQL data in a relational database.

Evaluation results show that the approach produces promising results. For instance when we evaluated the internal cluster quality of the produced clusters, we obtained results of the Silhouette Coefficient of value that are above zero (0), indicating relatively good clustering. In addition, evaltuating the ranking phase of the algorithm using the Cosine Similarity measure indicated that only information with a similarity measure greater than zero (0) when compared to the user query were included in the final list of ranked information to be returned to the user. Generaly, our evalution indicated that 
the developed multilevel text clustering approach was effective in the retrieval of academic research data.

The future work of this research lies in the choice of the number of clusters that the developed multilevel approach will produce. In our experiments, the number of clusters was chosen based on prior knowledge of the consolidated data in the repository used. As the amount and diversity of the data in the repository continues to increase, choosing the number of cluster this way may prove inefficient because it becomes difficult to manually go through all the data so as to determine the number of clusters to produce. Research can therefore explore a technique to automatically choose the optimal number of clusters based on the data to be clustered and embed it onto the algorithm for efficient clustering.

\section{ACKNOWLEDGEMENTS}

I want to express my gratitude to my employer Jomo Kenyatta University of Agriculture and Technology for sponsoring my studies and supporting this research work.

\section{REFERENCES}

[1] Muriithi, M. P. 2013. Computer Mediated Collaboration among the Academic Research Community: A Case Study of Kenya: Doctoral consortium paper. IEEE 7th International Conference on Research Challenges in Information Science (RCIS), 28-31 ${ }^{\text {st }}$ May 2013, Paris. DOI: https://doi.org/10.1109/RCIS.2013.6577731.

[2] Xia, F., Wang, W., Bekele, T. M., and Liu, H. 2017. Big Scholarly Data: A Survey. IEEE Transactions on Big Data, $\quad 3(1), \quad 18-35$. DOI: https://doi.org/10.1109/TBDATA.2016.2641460.

[3] Liu, J., Tang, T., Wang, W., Xu, B., and Kong, X. 2018. A Survey of Scholarly Data Visualization. IEEE Access, 6, 19205 - 19221. DOI: https://doi.org/10.1109/ACCESS.2018.2815030.

[4] Muriithi P.M 2015. Academic Research Collaborations in Kenya: Structure, Processes and Information Technologies (Unpublished doctoral dissertation). University of Brighton, United Kingdom.

[5] Mugambi, W.C., and Ongus, W.R. 2016. Analysis of the Implementation of an Institutional Repository: A Case Study of Dedan Kimathi University of Technology, Kenya. In International Journal of Information and Communication Studies, 2(1), 22-30.

[6] Erima, J., Masai, W., and Wosyanju, M, G. 2016. Preservation of Digital Research Content in Academic Institutions: A Case Study of Moi University, Kenya. In 2016 IST-Africa Week Conference, 11-13th May 2016, Durban, South Africa. DOI: https://doi.org/10.1109/ISTAFRICA.2016.7530620.

[7] Muriithi, P., Okeyo, G., and Waema, D. 2017. Towards improved availability and access to research data: A web based solution for Kenya. $12^{\text {th }}$ JKUAT Scientific Conference, Nairobi, 16-17 $7^{\text {th }}$ November 2017.

[8] Manju, K., Amita, J., Sonakshi, V., and Manoj, K. 2016. Analysis of Various Information Retrieval Models. In 3rd International Conference on Computing for Sustainable Global Development (INDIACom), 16-18 ${ }^{\text {th }}$ March 2016, New Delhi, India.

[9] Goker, A., and Davies, J. 2009. Information retrieval: Searching in the $21^{\text {st }}$ Century. Hoboken: John Wiley and Sons, Ltd.

[10] Desai, S. S., \& Laxminarayana, J. A. 2016. A Review of Semantic Based Techniques for Document Clustering. International Journal of Emerging Technology in Computer Science \& Electronics (IJETCSE), 22(2), 214 -217 .
[11] Namratha, M., and Prajwala, T. R. 2012. A Comprehensive Overview of Clustering Algorithms in Pattern Recognition. IOSR Journal of Computer Engineering (IOSRJCE), 4(6), 23-30. DOI: 10.9790/0661-0462330.

[12] Banerjee, S., Choudhary, A., and Pal, S. 2015. Empirical Evaluation of K-Means, Bisecting KMeans, Fuzzy C-Means and Genetic K-Means Clustering Algorithms. In 2015 IEEE International WIE Conference on Electrical and Computer Engineering (WIECON-ECE), 19-20 December 2015, Dhaka, Bangladesh. 168-172. DOI https://doi.org/10.1109/WIECON-ECE.2015.7443889.

[13] Jain, A., Bajpai, A., and Kumar R. M. 2012. Efficient Clustering Technique for Information Retrieval in Data Mining. International Journal of Emerging Technology and Advanced Engineering, 2(6), 12-20

[14] Hand, D. J., H. Mannila, H., and Smyth, P. 2001. Principles of data mining. MITPress, Cambridge, MA, USA. ISBN 0-262-08290-X.

[15] Ordonez, C. 2006. Integrating K-means Clustering with a Relational DBMS using SQL. IEEE Transactions on Knowledge and Data Engineering, 18(2), 188 - 201. DOI: https://doi.org/10.1109/TKDE.2006.31.

[16] Matusevich, S.D., and Ordonez, C. 2014. A Clustering Algorithm Merging MCMC and EM Methods Using SQL Queries. JMLR: Workshop and Conference Proceedings, 36, 61-76.

[17] Sun, Q., Fu, L., Deng, B., Pei, X., and Sun, J. 2017. An Efficient Distributed Database Clustering Algorithm for Big Data Processing. In 2017 3rd International Conference on Computational Systems and Communications CSP, $25-26^{\text {th }}$ March 2017, Beijing, China. DOI: 10.23977/iccsc.2017.1012.

[18] Manning, D.C., Raghavan, P., and Schütze, H. 2009. An introduction to information retrieval. Cambridge, UK: Cambridge University Press.

[19] Khan, S., Liu, X., Shakil, K.A., and Alam, M. 2017. A survey on scholarly data: From big data perspective. Information Processing and Management, 53(4), 923944. DOI: http://dx.doi.org/10.1016/j.ipm.2017.03.006.

[20] Saggion, H., and Ronzano, F. 2017. Scholarly Data Mining: Making Sense of Scientific Literature. In 2017 ACM/IEEE Joint Conference on Digital Libraries (JCDL), 19-23 June 2017, Toronto, ON, Canada. DOI: https://doi.org/10.1109/JCDL.2017.7991622.

[21] Sumba, X., Sumba, F., Tello, A., Baculima, F., Espinoza, M., and Saquicela, V. 2016. Detecting Similar Areas of Knowledge Using Semantic and Data Mining Technologies. Electronic Notes in Theoretical Computer Science, 329, 149-167. DOI: http://dx.doi.org/10.1016/j.entcs.2016.12.009.

[22] Katsurai, M. 2017. Bursty research topic detection from scholarly data using dynamic Co-word networks: A preliminary investigation. In 2017 IEEE 2nd International Conference on Big Data Analysis (ICBDA), 10-12 $2^{\text {th }}$ March 2017, Beijing, China. DOI: https://doi.org/10.1109/ICBDA.2017.8078788.

[23] Cormode, G., Muthukrishnan, S., and Yan, J. 2014. People like us: Mining scholarly data for comparable researchers. In Proceedings of the 23rd International Conference on World Wide Web, $07-11^{\text {th }}$, April 2014, Seoul, Korea.

DOI: https://doi.org/10.1145/2567948.2579038.

[24] Muia, M.A., and Oringo, J. 2016. Constraints on Research Productivity in Kenyan Universities: Case Study of University Of Nairobi, Kenya. International 
International Journal of Computer Applications Technology and Research

Volume 8-Issue 03, 66-81, 2019, ISSN:-2319-8656

Journal of Recent Advances in Multidisciplinary Research, 03 (8), 1785-1794.

[25] Liu, Y., Li, Z., Xiong, H., Gao, X., and Wu, J. 2010. Understanding of Internal Clustering Validation Measures. In IEEE International Conference on Data
Mining, 13-17 Dec. 2010, Sydney, NSW, Australia. DOI: https://doi.org/10.1109/ICDM.2010.35. 